\title{
Protocol Optimization for Surface Sterilization of Sugarcane Variety (B-52/298)
}

\author{
Yonas Zeslase Belete*, Hagos Kalu \\ Department of Biological and Chemical Engineering, MIT, Mekelle University, (TR), Ethiopia \\ *E-mail address: yonbelete7@gmail.com
}

\begin{abstract}
The aim of this study was to optimize conditions for surface sterilization of ex-plants of B52/298 sugarcane cultivar variety. Experiment was carried out to determine the optimum concentrations of surface sterilizing agents and at what time they optimally sterilize. This experiment was conducted at MIT Plant Tissue Culture and Micro-propagation Laboratory Center, situated in Mekelle City. Three chemisterilants (Sodium Hypochlorite, Mercuric Chloride and Ethanol) were taken for the investigation. Three of the sterilizing agents were used in different concentrations at various time intervals and each fungal and bacterial contamination was evaluated in intervals of three days from the initiation time for two weeks. The numbers of each contamination, clean cultures and dead ones were counted. Most of the contaminations were found to be fungal, while there was considerable numbers of cultures that were of bacterial contamination. Only a few of the cultures were observed as dead. Among these sterilizing chemicals, $60 \%$ Ethanol was found to be a better sterilizing agent when acted on the ex-plants for 6.5 minutes, resulting in $33.3 \%$ clean cultures.
\end{abstract}

Keywords: Sterilization; Chemisterilant; Ex-plant; Culture

\section{INTRODUCTION}

Do you know that an ineffective surface sterilization process always leads to bankruptcy for companies that deal with mass production of sugarcane? Which intern leads to shortage of raw materials to the sugar industries with which they have dealt with to supply? Such a big problem is only solved when an optimum surface sterilization process is used (Pande and Awasthi, 1996).

Sugarcane (accharum officinarum.L), which belongs to Poaceae family, is a tropical grass of high polyploidy ( $2 n=36-170)$. Sugarcane accounts for approximately $70 \%$ of the world's sugar and is an economically important cash crop in tropical and sub-tropical region of many countries (Chatenet et al., 2001). Properties such as an efficient photosynthesis and efficient biomass production make this an excellent target for industrial processing and a valuable alternative for animal feeding and the production of by-products. It is also a prime candidate as a fuel crop (production of ethanol) because of its efficient biomass production. Some other by-products from sugarcane include Molasses, Stock feed, Alcoholic drinks; Bagasse's and cane wax etc. (Gallo-Meagher et al., 2000).

Sugarcane is a clonally propagated crop from which multiple annual cuttings of stalks are typically obtained from each planting. This crop is especially vulnerable to diseases and propagation from cuttings facilitates the spread of pathogens and may results in epidemics (Schenck \& Lehrer, 2000). Sugarcane stalks can be infected by various pathogens without 
exhibiting any symptoms, and therefore there is a high risk of disease transfer during exchange and transport of sugarcane cuttings. Its growth is closely related to temperature. Optimum temperature for sprouting (germination) of stem cuttings is $32^{\circ} \mathrm{c}$ to $38^{\circ} \mathrm{c}$. It slows down below $25^{\circ} \mathrm{c}$, reaches plateau between $30^{\circ} \mathrm{c}-34^{\circ} \mathrm{c}$, is reduced above $35^{\circ} \mathrm{c}$ and practically stops when the temperature is above $38^{\circ}$. Temperatures above $38^{\circ}$ reduce the rate of photosynthesis and increase respiration. For ripening, however, relatively low temperatures in the range of $12^{\circ} \mathrm{c}$ to $14^{\circ} \mathrm{c}$ are desirable, since this has a noticeable influence on the reduction of vegetative growth rate and enrichment of sucrose in the cane. At higher temperatures, reversion of sucrose into fructose and glucose may occur besides enhancement of photorespiration thus leading to less accumulation of sugars. Severe cold weather inhibits bud sprouting in ratoon crop and arrests cane growth. Temperatures lower than $0^{\circ} \mathrm{C}$ induces freezing of less protected parts such as young leaves and lateral buds. The damage depends upon the length of the cold period. Smut initiation and spread is high at ambient temperatures of $25^{\circ} \mathrm{c}-30^{\circ} \mathrm{c}$. Similarly, the spread of red not disease is high at higher temperatures $\left(37^{\circ} \mathrm{c}-\right.$ $400^{\circ} \mathrm{c}$ ) when all other conditions are similar. Rust incidence is high when the minimum temperatures are drastically reduced. Shoot-fly incidence is high in summer when the air temperatures are very high. Also higher shoot fly incidence was observed when the difference between maximum (day) and minimum (night) temperature are low (Parmessur et al., 2002).

Ethiopia is endowed with favorable climate, enormous land and water resources for large scale irrigated development of sugarcane. The Federal Democratic Republic of Ethiopia, in its growth and development plan (GTP), has launched sugar development program to undertake new and expansion projects across the country with a clear objective of boosting sugar production to satisfy the domestic sugar demand as well as for any possible export. The government intends that Ethiopia should be self-sufficient in sugar by the end of 2013 and increase production almost eightfold to 2.3 million tonnes by mid-2015, leaving a surplus for export of 1.25 million tonnes, making the country one of the world's ten biggest exporters (Anonymous, 2013).

Recently, plant biotechnology and molecular biology have created unprecedented opportunities and promises in the field of agriculture. The spectular findings in plant tissue culture have generated outstanding interest, enthusiasm and optimism over the world. Methods have been developed for the propagation and efficient regeneration of many crops/plants through micro propagation. In order to ensure highest possible yield and quality of field crops the disease free stock can be extended to the growers by using this technique. However, there has to be sufficiently optimized protocol for the surface sterilization of the explant so that the required micro propagation will be achieved (Anonymous, 2009).

Plants brought from the field have high number of contamination on their surfaces. These contaminants will compete with the in vitro plants for nutrients if they are not removed. Mortality of the in vitro cultures increases because of microbial contamination and also shows the reduced growth and necrosis of cultures. Although B-52/298 sugarcane variety has a good yield and sugar content, its initiation for aseptic culture was found difficult. Using a combination of $20 \%$ Sodium Hypochlorite and $0.1 \%$ Mercuric Chloride running in the experiment for 10 minutes, MIT Plant Tissue Culture and Micro-propagation Laboratory Center found a clean culture of average of $20 \%$. Thus, looking for a better optimization is mandatory.

Use of unsterilized explants will result in loses of significant number of cultures. Due to the prevalence of high contaminants, achieving the goals the laboratory center plans each year is being highly tempted. The overall effect, besides to bogging down the progress of the tissue culture laboratory, it will have a direct impact on the sugar factories, which the laboratory center has dealt to supply the sugarcane annually. Consequently, it will result in an imbalance of sugar supply to customers. 
This contamination problem is becoming headache for the tissue culture laboratory. Though it has sufficient number of labor and well-equipped laboratory equipment, elimination of contamination to the level they cannot hinder the initiation and growth of cultivars has been the most challenging work. This will only be solved when an optimum protocol is established for the elimination of bacteria and fungi on the surface of the explants.

The main goal of this research was to optimize surface sterilization method for B$52 / 298$ sugarcane variety.

This experimental work would enable to meet the specific objectives for the B-52/298 sugarcane variety for determine the optimum concentrations of surface sterilizing agents and determine effective time for sterilization.

\section{MATERIALS AND METHODOLOGY}

\subsection{Geographical Location}

This experiment was conducted at MIT Plant Tissue Culture and Micro-propagation Laboratory Center, situated in Mekelle city, which is located at latitude and longitude of $13^{\circ} 29^{\prime} \mathrm{N}$ and $39^{\circ} 28^{\prime}$ E respectively.

\subsection{Materials and Chemicals Used}

The following materials and chemicals were used in the experimental study, though the list may not be inclusive.

\begin{tabular}{|c|c|c|}
\hline 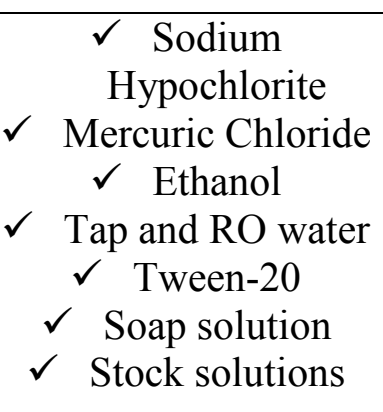 & $\begin{array}{cc}\checkmark & \text { Sugar ex-plants } \\
\checkmark & \text { Blades } \\
\checkmark & \text { Forceps } \\
\checkmark & \text { Scalpels } \\
\checkmark & \text { PH meter } \\
\checkmark & \text { Balance } \\
\checkmark & \text { Glass bead sterilizer }\end{array}$ & $\begin{array}{cc}\checkmark & \text { Plate } \\
\checkmark & \text { Towel } \\
\checkmark & \text { Trays } \\
\checkmark & \text { Autoclave } \\
\checkmark & \text { Gloves } \\
\checkmark & \text { Flasks }\end{array}$ \\
\hline
\end{tabular}

\subsection{Procedure}

Every laboratory facilities were provided by MIT Plant Tissue Culture and Micropropagation Laboratory Center where all tasks of the experimental work were carried out.

A specific sugarcane variety, B-52/298, was used for our investigation. This ex-plant material was taken from a one-month-old sugarcane plant. While excising the ex-plants, the outer sheath was removed until the apical meristem at the tip was found. They were thoroughly washed under running tape water followed by soap solution for two minutes. Cutting them into small pieces, the outer mature parts were removed until a spindled of about $2 \mathrm{~cm}$ in diameter was obtained. Then they were soaked in a $0.25 \mathrm{~g}$ Bayluton, $0.25 \mathrm{~g}$ Redomil and shaken for 30 minutes. The explants were again washed with sterile distilled water for 10 minutes and then taken inside the laminar hood for further sterilization. Soaking each spindle in the specified concentrations of each sterilizing agents, they were shaken well until the due time. 
For this experimental work, three sterilizing chemicals (Mercuric Chloride, Sodium Hypochlorite, and Ethanol) were used at various concentrations for different time of exposures as the tables below shown.

Table 1. Surface Sterilization of Sugarcane Using Mercuric Chloride.

\begin{tabular}{|c|c|c|c|c|}
\hline Variety & $\begin{array}{c}\text { Treatment } \\
\text { number }\end{array}$ & $\begin{array}{c}\text { Concentration used } \\
(\%)\end{array}$ & Time (in minutes) & Replication \\
\hline \multirow{9}{*}{ B-52/298 } & \multirow{3}{*}{ T1 } & 0.1 & 10 & \multirow{3}{*}{$R=4$} \\
\hline & & 0.1 & 20 & \\
\hline & & 0.1 & 30 & \\
\hline & \multirow{3}{*}{$\mathbf{T 2}$} & 0.125 & 10 & \multirow{3}{*}{$R=4$} \\
\hline & & 0.125 & 15 & \\
\hline & & 0.125 & 20 & \\
\hline & \multirow{3}{*}{ T3 } & 0.25 & 5 & \multirow{3}{*}{$R=4$} \\
\hline & & 0.25 & 10 & \\
\hline & & 0.25 & 15 & \\
\hline
\end{tabular}

Table 2. Surface Sterilization of Sugarcane Using Sodium Hypochlorite.

\begin{tabular}{|c|c|c|c|c|}
\hline Variety & $\begin{array}{c}\text { Treatment } \\
\text { number }\end{array}$ & $\begin{array}{c}\text { Concentration used } \\
(\%)\end{array}$ & Time(in minutes) & Replication \\
\hline \multirow{9}{*}{ B-52/298 } & \multirow{3}{*}{ T1 } & 15 & 20 & \multirow{3}{*}{$R=4$} \\
\hline & & 15 & 30 & \\
\hline & & 15 & 40 & \\
\hline & \multirow{3}{*}{$\mathbf{T} 2$} & 20 & 10 & \multirow{3}{*}{$R=4$} \\
\hline & & 20 & 20 & \\
\hline & & 20 & 30 & \\
\hline & \multirow{3}{*}{ T3 } & 30 & 5 & \multirow{3}{*}{$R=4$} \\
\hline & & 30 & 10 & \\
\hline & & 30 & 15 & \\
\hline
\end{tabular}

Table 3. Surface Sterilization of Sugarcane Using Ethanol.

\begin{tabular}{|c|c|c|c|c|}
\hline Variety & $\begin{array}{l}\text { Treatment } \\
\text { number }\end{array}$ & $\begin{array}{c}\text { Concentration used } \\
(\%)\end{array}$ & $\begin{array}{c}\text { Time } \\
\text { (in minutes) }\end{array}$ & Replication \\
\hline \multirow{9}{*}{ B-52/298 } & \multirow{3}{*}{ T1 } & 60 & 2.50 & \multirow{3}{*}{$R=4$} \\
\hline & & 60 & 4.50 & \\
\hline & & 60 & 6.50 & \\
\hline & \multirow{3}{*}{$\mathbf{T 2}$} & 70 & 1.00 & \multirow{3}{*}{$R=4$} \\
\hline & & 70 & 2.00 & \\
\hline & & 70 & 3.00 & \\
\hline & \multirow{3}{*}{ T3 } & 80 & 0.50 & \multirow{3}{*}{$R=4$} \\
\hline & & 80 & 1.00 & \\
\hline & & 80 & 1.50 & \\
\hline
\end{tabular}


Prior to inoculation, the ex-plants were rinsed with RO water to remove any traces of the sterilizing chemicals used.

\section{RESULTS}

Each fungal and bacterial contamination was evaluated in intervals of the three days from the initiation time for two weeks. The numbers for each contamination, clean cultures and dead ones were counted. For each of the sterilizing chemicals, the result was found as the following tables shown.

Table 4. Result of Surface Sterilization of Sugarcane Using Mercuric Chloride.

\begin{tabular}{|c|c|c|c|c|c|c|c|}
\hline Variety & $\begin{array}{c}\text { Treatment } \\
\text { number }\end{array}$ & 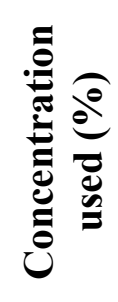 & $\begin{array}{l}\text { Time (in } \\
\text { minutes) }\end{array}$ & 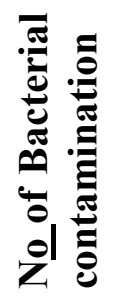 & $\begin{array}{l}\text { No of Fungal } \\
\text { Contamination }\end{array}$ & 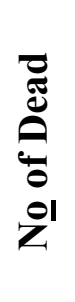 & $\begin{array}{l}\text { No of } \\
\text { Clean }\end{array}$ \\
\hline \multirow{12}{*}{$\begin{array}{c}\text { B- } \\
52 / 298\end{array}$} & \multirow{4}{*}{$\mathbf{T 1}$} & 0.1 & 10 & 1 & 4 & $\mathbf{0}$ & $\mathbf{0}$ \\
\hline & & 0.1 & 20 & 1 & 2 & $\mathbf{0}$ & $\mathbf{0}$ \\
\hline & & 0.1 & 30 & 0 & 2 & 2 & $\mathbf{0}$ \\
\hline & & Total & & 2 & 8 & 2 & $\mathbf{0}$ \\
\hline & \multirow{4}{*}{$\mathbf{T 2}$} & 0.125 & 10 & 0 & 2 & $\mathbf{0}$ & 1 \\
\hline & & 0.125 & 15 & $\mathbf{0}$ & 4 & $\mathbf{0}$ & 0 \\
\hline & & 0.125 & 20 & 0 & 3 & 1 & 1 \\
\hline & & Total & & $\mathbf{0}$ & 9 & 1 & 2 \\
\hline & \multirow{4}{*}{ T3 } & 0.25 & 5 & $\mathbf{0}$ & 2 & $\mathbf{0}$ & 1 \\
\hline & & 0.25 & 10 & 2 & 1 & $\mathbf{0}$ & $\mathbf{0}$ \\
\hline & & 0.25 & 15 & $\mathbf{0}$ & 2 & $\mathbf{0}$ & 1 \\
\hline & & Total & & 2 & 5 & $\mathbf{0}$ & 2 \\
\hline
\end{tabular}


Table 5. Result of Surface Sterilization of Sugarcane Using Sodium Hypochlorite.

\begin{tabular}{|c|c|c|c|c|c|c|c|}
\hline Variety & $\begin{array}{c}\text { Treatment } \\
\text { number }\end{array}$ & 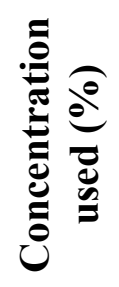 & $\begin{array}{l}\text { Time (in } \\
\text { minutes) }\end{array}$ & 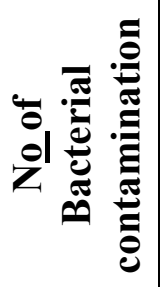 & $\begin{array}{l}\text { No of Fungal } \\
\text { Contamination }\end{array}$ & $\begin{array}{l}\text { No of } \\
\text { Dead }\end{array}$ & $\begin{array}{l}\text { No of } \\
\text { Clean }\end{array}$ \\
\hline \multirow{12}{*}{$\begin{array}{c}\text { B- } \\
52 / 298\end{array}$} & \multirow{4}{*}{ T1 } & 15 & 20 & 1 & 2 & $\mathbf{0}$ & 1 \\
\hline & & 15 & 30 & 1 & 3 & $\mathbf{0}$ & $\mathbf{0}$ \\
\hline & & 15 & 40 & 1 & 2 & $\mathbf{0}$ & 1 \\
\hline & & Total & & 3 & 7 & $\mathbf{0}$ & 2 \\
\hline & \multirow{4}{*}{$\mathbf{T} 2$} & 20 & 10 & 1 & 3 & $\mathbf{0}$ & $\mathbf{0}$ \\
\hline & & 20 & 20 & 1 & 3 & $\mathbf{0}$ & $\mathbf{0}$ \\
\hline & & 20 & 30 & 1 & 3 & $\mathbf{0}$ & $\mathbf{0}$ \\
\hline & & Total & & 3 & 9 & $\mathbf{0}$ & $\mathbf{0}$ \\
\hline & \multirow{4}{*}{ T3 } & 30 & 5 & 2 & 2 & $\mathbf{0}$ & $\mathbf{0}$ \\
\hline & & 30 & 10 & 3 & $\mathbf{0}$ & $\mathbf{0}$ & 1 \\
\hline & & 30 & 15 & 1 & 1 & 1 & 1 \\
\hline & & Total & & 6 & 3 & 1 & 2 \\
\hline
\end{tabular}

Table 6. Result of Surface Sterilization of Sugarcane Using Ethanol.

\begin{tabular}{|c|c|c|c|c|c|c|c|}
\hline Variety & $\begin{array}{c}\text { Treatment } \\
\text { number }\end{array}$ & 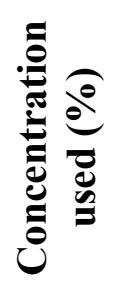 & $\begin{array}{l}\text { Time (in } \\
\text { minutes) }\end{array}$ & 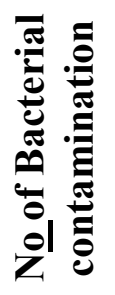 & $\begin{array}{c}\text { № of Fungal } \\
\text { Contamination }\end{array}$ & $\begin{array}{l}\text { No of } \\
\text { Dead }\end{array}$ & $\begin{array}{l}\text { No of } \\
\text { Clean }\end{array}$ \\
\hline \multirow{12}{*}{$\begin{array}{c}\text { B- } \\
52 / 298\end{array}$} & \multirow{4}{*}{ T1 } & 60 & 2.5 & 1 & 2 & $\mathbf{0}$ & 1 \\
\hline & & 60 & 4.5 & 2 & 1 & $\mathbf{0}$ & 1 \\
\hline & & 60 & 6.5 & 1 & $\mathbf{0}$ & $\mathbf{0}$ & 2 \\
\hline & & Total & & 4 & 3 & $\mathbf{0}$ & 4 \\
\hline & \multirow{4}{*}{$\mathbf{T 2}$} & 70 & 1 & 1 & 1 & $\mathbf{0}$ & 2 \\
\hline & & 70 & 2 & 3 & 1 & $\mathbf{0}$ & $\mathbf{0}$ \\
\hline & & 70 & 3 & 1 & 3 & $\mathbf{0}$ & $\mathbf{0}$ \\
\hline & & Total & & 5 & 5 & $\mathbf{0}$ & 2 \\
\hline & \multirow{4}{*}{ T3 } & 80 & 0.5 & 2 & 2 & $\mathbf{0}$ & $\mathbf{0}$ \\
\hline & & 80 & 1 & 1 & 2 & $\mathbf{0}$ & 1 \\
\hline & & 80 & 1.5 & 5 & 0 & 0 & $\mathbf{0}$ \\
\hline & & Total & & 8 & 4 & 0 & 1 \\
\hline
\end{tabular}




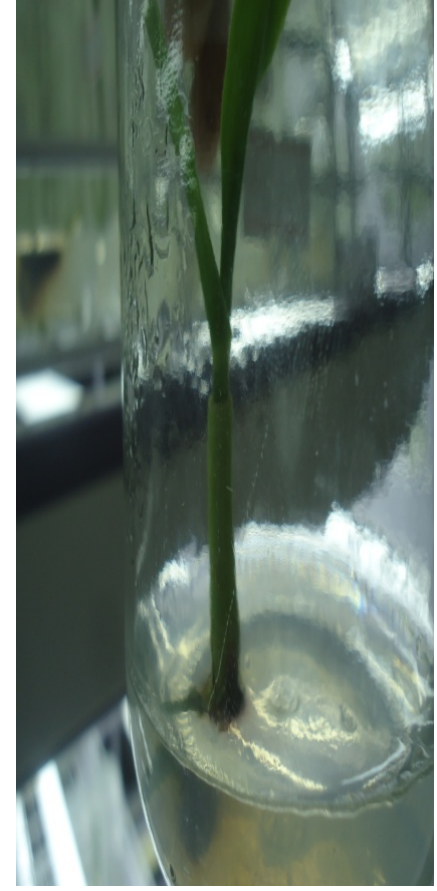

Figure 1. Clean Culture.

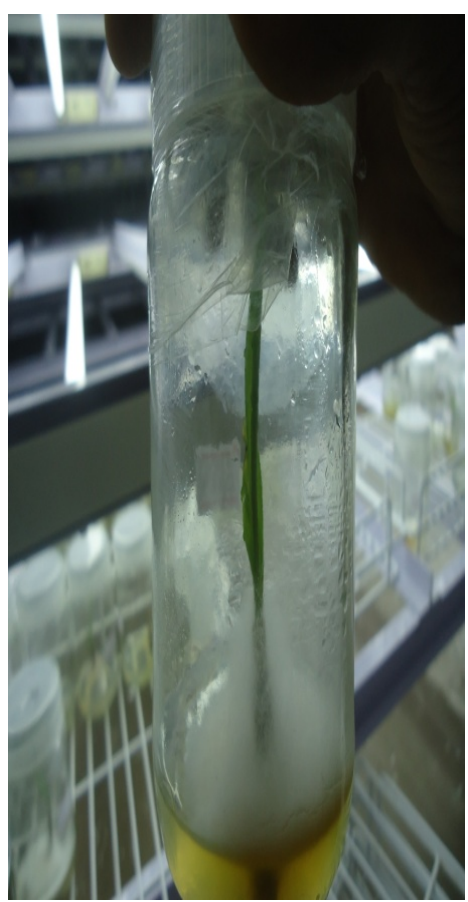

Figure 2. Fungi.

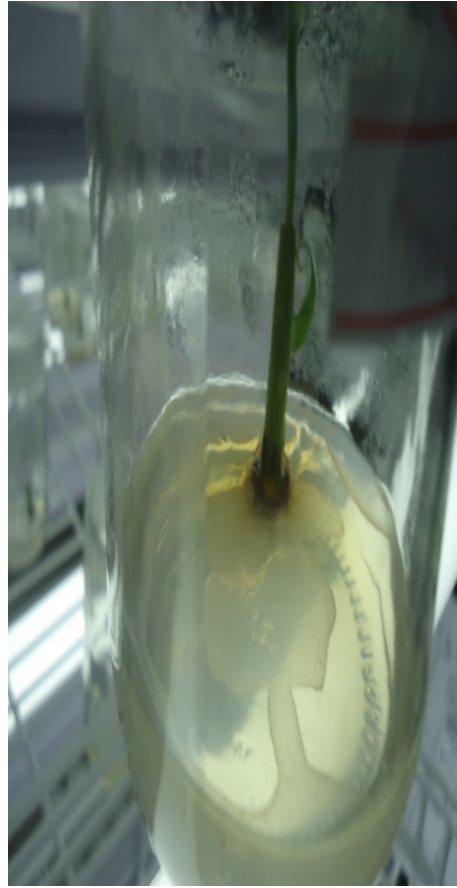

Figure 3. Bacteria.

\section{DATA ANALYSIS AND INTERPRETATIONS}

Table 7. Analysis of Surface Sterilization of Sugarcane Using Mercuric Chloride.

\begin{tabular}{|c|c|c|c|c|c|c|c|c|c|c|c|}
\hline \multirow[t]{2}{*}{ Variety } & \multirow[t]{2}{*}{$\begin{array}{c}\text { Treatment } \\
\text { number }\end{array}$} & \multirow[t]{2}{*}{ 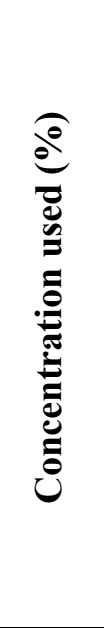 } & \multirow[t]{2}{*}{$\begin{array}{l}\text { Time (in } \\
\text { minutes) }\end{array}$} & \multicolumn{2}{|c|}{ 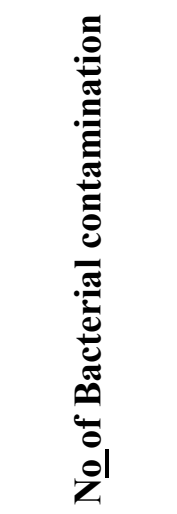 } & \multicolumn{2}{|c|}{ 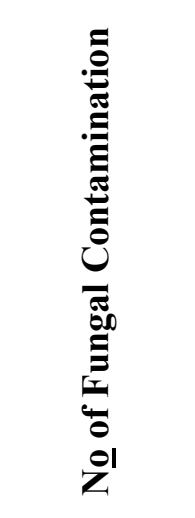 } & \multicolumn{2}{|c|}{ № of Dead } & \multicolumn{2}{|c|}{$\begin{array}{l}\text { No of } \\
\text { Clean }\end{array}$} \\
\hline & & & & No & $\%$ & No & $\%$ & No & $\%$ & $\begin{array}{l}\mathbf{N} \\
\underline{\mathbf{0}}\end{array}$ & $\%$ \\
\hline \multirow{12}{*}{$\begin{array}{c}\text { B- } \\
52 / 298\end{array}$} & \multirow{4}{*}{ T1 } & 0.1 & 10 & 1 & 20 & 4 & 80 & $\mathbf{0}$ & & $\overline{\mathbf{0}}$ & \\
\hline & & 0.1 & 20 & 1 & 33.3 & 2 & 66.6 & $\mathbf{0}$ & & $\mathbf{0}$ & \\
\hline & & 0.1 & 30 & $\mathbf{0}$ & $\mathbf{0}$ & 2 & 50 & $2 y$ & 50 & $\mathbf{0}$ & \\
\hline & & Total & & 2 & 16.6 & 8 & 66.6 & 2 & 16.6 & ( & 0 \\
\hline & \multirow{4}{*}{$\mathrm{T} 2$} & 0.125 & 10 & $\mathbf{0}$ & & 2 & & $\mathbf{0}$ & & 1 & \\
\hline & & 0.125 & 15 & $\mathbf{0}$ & & 4 & & $\mathbf{0}$ & & 0 & \\
\hline & & 0.125 & 20 & (0 & & 3 & & 1 & & 1 & \\
\hline & & Total & & $\mathbf{0}$ & $\mathbf{0}$ & 9 & 75 & 1 & 8.3 & 2 & 16.6 \\
\hline & \multirow{4}{*}{ T3 } & 0.25 & 5 & $\mathbf{0}$ & & 2 & & $\mathbf{0}$ & & 1 & \\
\hline & & 0.25 & 10 & 2 & & 1 & & $\mathbf{0}$ & & $\mathbf{0}$ & \\
\hline & & 0.25 & 15 & $\mathbf{0}$ & & 2 & & $\mathbf{0}$ & & 1 & \\
\hline & & Total & & 2 & 16.6 & 5 & 41.6 & $\mathbf{0}$ & $\mathbf{0}$ & 2 & 16.6 \\
\hline
\end{tabular}


As table 7 shows, the second and third treatments $(0.125 \%$ and $0.25 \%$ of Mercuric Chloride) have resulted in some clean cultures (16.6\% in both cases). In T2, most of the contaminations were fungal $(75 \%)$ while there was no contamination observed because of bacteria. Some dead cultures were also observed (8.3\%).

Having observed in the same table, most of the contamination in the third treatment (T3) comes from fungi $(41.6 \%)$, but some bacterial contaminations (16.6\%) were also observed. Fortunately, no dead culture was found.

Table 8. Analysis of Surface Sterilization of Sugarcane Using Sodium Hypochlorite.

\begin{tabular}{|c|c|c|c|c|c|c|c|c|c|c|c|}
\hline \multirow[t]{2}{*}{ Variety } & \multirow[t]{2}{*}{$\begin{array}{c}\text { Treatment } \\
\text { number }\end{array}$} & \multirow[t]{2}{*}{ 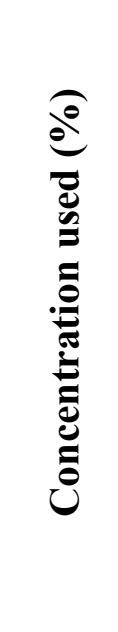 } & \multirow[t]{2}{*}{$\begin{array}{c}\text { Time } \\
\text { (in } \\
\text { minutes) }\end{array}$} & \multicolumn{2}{|c|}{ 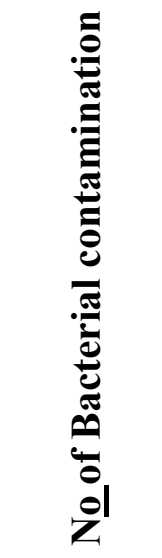 } & \multicolumn{2}{|c|}{ 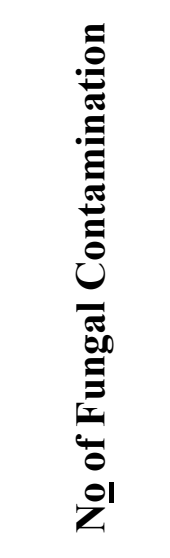 } & \multicolumn{2}{|c|}{$\begin{array}{l}\text { No of } \\
\text { Dead }\end{array}$} & \multicolumn{2}{|c|}{$\begin{array}{l}\text { No of } \\
\text { Clean }\end{array}$} \\
\hline & & & & No & $\%$ & No & $\%$ & No & $\%$ & No & $\%$ \\
\hline \multirow{12}{*}{$\begin{array}{c}\text { B- } \\
52 / 298\end{array}$} & \multirow{4}{*}{ T1 } & 15 & 20 & 1 & & 2 & & $\mathbf{0}$ & & 1 & \\
\hline & & 15 & 30 & 1 & & 3 & & $\mathbf{0}$ & & $\mathbf{0}$ & \\
\hline & & 15 & 40 & 1 & & 2 & & 0 & & 1 & \\
\hline & & Total & & 3 & 25 & 7 & 58 & $\mathbf{0}$ & $\mathbf{0}$ & 2 & 16.6 \\
\hline & \multirow{4}{*}{$\mathbf{T} 2$} & 20 & 10 & 1 & & 3 & & $\mathbf{0}$ & & $\mathbf{0}$ & \\
\hline & & 20 & 20 & 1 & & 3 & & $\mathbf{0}$ & & $\mathbf{0}$ & \\
\hline & & 20 & 30 & 1 & & 3 & & 0 & & $\mathbf{0}$ & \\
\hline & & Total & & 3 & 25 & 9 & 75 & $\mathbf{0}$ & & $\mathbf{0}$ & $\mathbf{0}$ \\
\hline & \multirow{4}{*}{ T3 } & 30 & 5 & 2 & & 2 & & $\mathbf{0}$ & & $\mathbf{0}$ & \\
\hline & & 30 & 10 & 3 & & $\mathbf{0}$ & & $\mathbf{0}$ & & 1 & \\
\hline & & 30 & 15 & 1 & & 1 & & 1 & & 1 & \\
\hline & & Total & & 6 & 50 & 3 & 25 & 1 & 8.3 & 2 & 16.6 \\
\hline
\end{tabular}

From table 8, we can see that some clean cultures (16.6\%) were observed in the first (T1) and third (T3) treatments which are of $15 \%$ and $30 \%$ concentrations of sodium hypochlorite solution respectively. The table also shows us that most of the contaminations in the first treatment $(58 \%)$ come from bacteria while the rest $(25 \%)$ is of fungal contamination. 
Table 9. Analysis of Surface Sterilization of Sugarcane Using Ethanol.

\begin{tabular}{|c|c|c|c|c|c|c|c|c|c|c|c|}
\hline \multirow[t]{2}{*}{ Variety } & \multirow[t]{2}{*}{$\begin{array}{c}\text { Treatment } \\
\text { number }\end{array}$} & \multirow{2}{*}{ 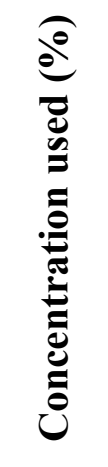 } & \multirow[t]{2}{*}{$\begin{array}{l}\text { Time (in } \\
\text { minutes) }\end{array}$} & \multicolumn{2}{|c|}{ 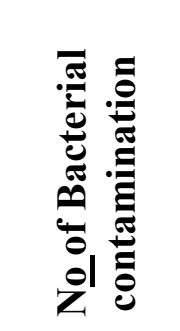 } & \multicolumn{2}{|c|}{ 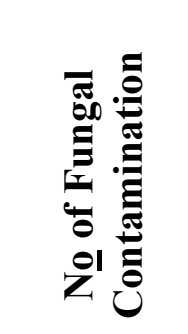 } & \multicolumn{2}{|c|}{$\begin{array}{l}\text { No of } \\
\text { Dead }\end{array}$} & \multicolumn{2}{|c|}{$\begin{array}{l}\text { No of } \\
\text { Clean }\end{array}$} \\
\hline & & & & No & $\%$ & No & $\%$ & No & $\%$ & No & $\%$ \\
\hline \multirow{12}{*}{$\begin{array}{c}\text { B- } \\
52 / 298\end{array}$} & \multirow{4}{*}{ T1 } & 60 & 2.5 & 1 & 25 & 2 & 50 & $\mathbf{0}$ & $\mathbf{0}$ & 1 & 25 \\
\hline & & 60 & 4.5 & 2 & 50 & 1 & 25 & $\mathbf{0}$ & $\mathbf{0}$ & 1 & 25 \\
\hline & & 60 & 6.5 & 1 & 25 & $\mathbf{0}$ & $\mathbf{0}$ & $\mathbf{0}$ & $\mathbf{0}$ & 2 & 50 \\
\hline & & Total & & 4 & 33.3 & 3 & 25 & $\mathbf{0}$ & $\mathbf{0}$ & 4 & 33.3 \\
\hline & & 70 & 1 & 1 & & 1 & & $\mathbf{0}$ & & 2 & \\
\hline & 12 & 70 & 2 & 3 & & 1 & & $\mathbf{0}$ & & $\mathbf{0}$ & \\
\hline & & 70 & 3 & 1 & & 3 & & $\mathbf{0}$ & & $\mathbf{0}$ & \\
\hline & & Total & & 5 & 41.6 & 5 & 41.6 & $\mathbf{0}$ & & 2 & 16.6 \\
\hline & & 80 & 0.5 & 2 & & 2 & & $\mathbf{0}$ & & $\mathbf{0}$ & \\
\hline & 13 & 80 & 1 & 1 & & 2 & & $\mathbf{0}$ & & 1 & \\
\hline & & 80 & 1.5 & 5 & & $\mathbf{0}$ & & $\mathbf{0}$ & & $\mathbf{0}$ & \\
\hline & & Total & & 8 & 61.6 & 4 & 30.3 & $\mathbf{0}$ & & 1 & 7.7 \\
\hline
\end{tabular}

As we can see from table 9, three of the treatments (T1, T2, and T3) using ethanol concentrations of $60 \%, 70 \%$ and $80 \%$ have come with clean cultures of $33.3 \%, 16.6 \%$, and $7.7 \%$ respectively when they are treated with their respective minutes as indicated in the table. The $60 \%$ concentration of ethanol with an exposure time of 6.5 minutes has resulted into half of the clean cultures while the rest half percentage is equally shared by the rest exposure times (2.5 minutes and 4.5 minutes). From the table, we can also observe that the $60 \%$ ethanol treatment has come with $33.3 \%$ of bacterial and $25 \%$ fungal contaminations.

Bacterial and fungal contaminations of $41.6 \%$ were also found in the second treatment (70\% ethanol concentration), while majority of the contaminations $(61.6 \%)$ were observed to be that of bacterial contamination. The rest contamination $(30.3 \%)$ was found to be that of fungal. The results of this experiment have some significance in that about $13 \%$ of cultures per one cycle of sterilization would have been saved. The other very important point that makes this finding facilitating is that ethanol is relatively cheaper and easily available. However, even the findings of this experiment are not that satisfactory in comparison to the results recorded in different literatures. Gosal et al, 1998, has described surface sterilization of 
explants with $95 \%$ ethanol for 5 minutes and found $45 \%$ clean cultures whereas Nand, L., 1997 , used $65 \%$ ethanol for 10 minutes and reported that he found $67 \%$ clean cultures.

\section{CONCLUSION}

From the analyzed data, the following conclusions have been made: Mercuric Chloride is a better chemisterilant when it is diluted to either $0.125 \%$ or $0.25 \%$ concentrations, with the result about $17 \%$ clean cultures in both cases, while Sodium Hypochlorite acts more effectively in the concentrations of $15 \%$ and $30 \%$ resulting into the same number of clean cultures as Mercuric Chloride. Concerning to the chemisterilant ethanol $60 \%$ of the used concentrations has been found being more effective sterilizing agent, whose result of clean culture was recorded to be around $33 \%$.

Thus, ethanol is the best sterilizing agent. This chemical acted more efficiently when it was applied in $60 \%$ when the ex-plants were exposed for 6.5 minutes. In addition, the other thing we could deduce from this experimental study is that the external surface sterilization is not that optimum.

\section{Acknowledgment}

We wholeheartedly would like to acknowledge all those persons who one way or another assisted us in the completion of this study. To us, it is a proud and privilege to specifically ventilate deep sense of gratitude and acclamation to our advisors Mr. Jemal Aman, the Project Manager of MIT (Mekelle Institute of Technology) Plant Tissue Culture and Micro-propagation Laboratory, and our instructor Hailekiros Tadesse for their advice, supervision and encouragement. Without their guidance, constructive criticism and meticulous scrutiny, this work would not have seen light of the day.

Our heartfelt acknowledgment also goes to MIT Plant Tissue Culture and Micro-propagation Laboratory in general for giving us full access for their laboratory with its complete chemicals and instruments.

\section{References}

[1] Anonymous, (2009). Cetrel and Novozymes to Make Biogas and Electricity from Bagasse. Business Wire. 14 December 2009.

[2] Anonymous, (2013). http://www.mfa.gov.et/weekHornAfrica/morewha.php?869. As reviewed on $03 / 09 / 2013$

[3] Chatenet, M., C. Delage and M. Ripolles, (2001). Detection of sugarcane yellow leaf curl virus in quarantine and production of virus-free sugarcane by apical meristem culture. Plant Disease, 85(11): 1177-1180.

[4] Gallo-Meagher, M., R.G. English and A. Abouzid, (2000). Thidiazuron stimulates shoot regeneration of sugarcane embryogenic callus. In Vitro Cel lular and Developmental Biology Plant, 36: 37-40.

[5] Gosal, S.S., K.S. Thind and H.S. Dhaliwal, (1998). Micropropagation of sugarcane an efficient protocol for commercial plant production. Crop Impro., 25(2): 167-171.

[6] Lal, J., H.P. Pande and S.K. Awasthi, (1996). A general micro propagation protocol for sugarcane varieties. New Bot., 23(1/4):13-19. 
[7] Nand, L. and K. Ram. (1997). Yield comparison in sugarcane crop raised from conventional and mericlone derived seed cane. Ind. Sugar, 47(8): 617-621.

[8] Parmessur, Y., A. Aljanabi, S. Saumtally and A. Dookun-Saumtally, (2002). Sugarcane yellow leaf virus and sugarcane yellows phytoplasma: elimination by tissue culture. Plant Pathology, 51: 561-566.

[9] Schenck, S. and A.T. Lehrer. (2000). Factors affecting the transmission and spread of Sugarcane yellow leaf virus. Plant Disease, 84(10): 1085-1088. 